\title{
Wolfgang Pauli and the Fine-Structure Constant
}

\author{
Michael A. Sherbon \\ michael.sherbon@case.edu
}

September 17, 2012

\begin{abstract}
Wolfgang Pauli was influenced by Carl Jung and the Platonism of Arnold Sommerfeld, who introduced the fine-structure constant. Pauli's vision of a World Clock is related to the symbolic form of the Emerald Tablet of Hermes and Plato's geometric allegory otherwise known as the Cosmological Circle attributed to ancient tradition. With this vision Pauli revealed geometric clues to the mystery of the fine-structure constant that determines the strength of the electromagnetic interaction. A Platonic interpretation of the World Clock and the Cosmological Circle provides an explanation that includes the geometric structure of the pineal gland described by the golden ratio. In his experience of archetypal images Pauli encounters the synchronicity of events that contribute to his quest for physical symmetry relevant to the development of quantum electrodynamics.
\end{abstract}

\section{Introduction}

Wolfgang Pauli (1900-1958) was an Austrian-Swiss theoretical physicist noted for his work on the discovery of the exclusion principle, the fourth quantum number in the theory of spin, prediction of the neutrino, and calculation of the hydrogen spectrum [1]-[5]. While under the direction of Arnold Sommerfeld at the university, Pauli was influenced by Sommerfeld's search for a Platonic understanding of physics and connections implied by the mystery of the fine-structure constant. Pauli's interest in the philosophy of science was applied to the interpretation of quantum physics and magnified with the analysis of his dreams early in his career by Carl Jung [5]. This work then deepened his attraction toward the relationship between his dream images and the abstract concepts he encountered in physics. Richard Feynman proclaimed the fine-structure constant as one of the greatest mysteries of physics [2]. Physicist Max Born declared, "... the explanation of this number must be the central problem of natural philosophy." [2]. As the fine-structure constant determines the electromagnetic strength its theoretical origin was for Pauli "... the most important of the unsolved problems of ... physics." $[3,6]$. 


\section{Wolfgang Pauli's World Clock}

Both Pauli's waking vision of the World Clock and the Cosmological Circle embrace the archetypal images of space with the circle, square, triangle, and the cycles of time. In an allegorical study Plato described the geometric proportions of the Cosmological Circle $[7,8]$, the ground plan for many ancient monuments and temples. The Pythagorean geometry of the $3,4,5$ right triangle and the "squared circle" form the basis of the Cosmological Circle. The heptagon is an additional feature of the Cosmological Circle, and its relation to the cycloid curve connect it to the foundation of calculus and the history of the least action principle. Pauli's world clock has three rhythms with two orthogonal central discs, encircled by a gold colored band divided into 32 parts, and supported by a blackbird (the bird of Hermes [9]). The outer gold colored band represents 32 cubed or 32,768 total pulses of the three rhythms, which is the harmonic of Walter Russell's carbon pressure point of the carbon octave and significant to William Conner's harmonic system [10] related to the energy of the World Grid [11]. According to Pauli a feeling of "most sublime harmony" was produced by this abstract structure [2]. "According to Jung, Pauli's vision of the World Clock represents the essence of space-time ..." [1, 12], and was likened to Kepler's first geometrical ordering of the solar system, similar to the construction from the ancient canon that involves the geometry of the polygon circumscribing constant; to which Pauli adds his interpretation of time in his "Great Vision."

Another interpretation of Pauli's World Clock could be made comparing it to a basic yin-yang space-time model of brain-mind function describing hemispheric interactions [13]. Pauli associated the rhythms of the World Clock with biological processes (in particular the four chambers of the heart and its average rhythm of 72 beats per minute) as well as with psychic processes [14]. In Wolfgang Pauli's visionary World Clock geometry the blackbird is a symbol for the "turning inward" at the beginning stage of alchemy and the messenger for the creative solar principle. Corresponding to this inward turnabout is the angle of $180^{\circ}=32^{\circ}+\left(4 \times 37^{\circ}\right) \simeq 32^{\circ}+4 \tan ^{-1}(3 / 4)$. From the Beyers-Brown drawing of Pauli's dream, half of a golden rectangle encloses the blackbird. A line extended from the left side of the golden circle through the beak to the back foot, then a right angle up to the right side of the gold circle forms a triangle of approximately $32^{\circ}, 90^{\circ}$, and $58^{\circ}$ respectively. The $\cot 32^{\circ} \simeq \sec (2 \pi / 7) \simeq 16 / \pi^{2} \simeq \phi$, the golden ratio. The $\csc ^{2} 32^{\circ} \approx 1+\phi^{2}=\phi \sqrt{5}$, square of the diagonal of a golden rectangle, and $360^{\circ} / \phi^{2} \simeq 137.5^{\circ}$ the golden angle. $7 \phi$ divides the circle into approximately $32^{\circ}$ segments, $360^{\circ} / 7 \phi \simeq 32^{\circ}\left(\phi=2 \cos 36^{\circ} \simeq 1.618033988749\right.$, see Eq. (6)), $32^{\circ}+45^{\circ}=77^{\circ} \simeq 3 \pi / 7$ radians, apex angle of the large triangle in the heptagon. One radian, $360^{\circ} / 2 \pi \simeq 32^{\circ}+180^{\circ} / 7$.

The World Clock has three rhythms or pulses and 32 partitions dividing the circle, $360^{\circ} / 32=45^{\circ} / 4=\pi / 16$ radians and $\cos (\pi / 16) \approx R \cos 32^{\circ}$ where $R$ is the outer radius of the heptagon with side equal to one, $R \sin 32^{\circ} \simeq 3 / 5$. On the top of this "Pauli triangle" are two smaller back-to-back triangles with approximately the same angles. The apex at the top of the vertical disk is $58^{\circ}+58^{\circ} \simeq 116^{\circ}$, approximately the regular dodecahedron dihedral angle. The dihedral angle is the internal face angle where the two adjacent faces of the polyhedron meet. 


\section{Space, time, and synchronicity}

The subject of synchronicity was investigated at length by Pauli and Jung in their essay on The Interpretation of Nature and the Psyche [15] and the geometry of this artwork could be an example of a synchronistic "meaningful coincidence" as well as intuitive clues toward something more than "numerical coincidence" for calculations that follow [16]. The "Pauli triangle" is also found in the symbolic form of the Emerald Tablet of Hermes, appearing at the very end of the same chapter of Jung's description of Pauli's World Clock and having a vertex on the Philosopher's Stone [12]. The "Golden Chains of Homer extending from the Central Ring of Plato" [17] in the tablet divides the mandala into the golden angle and forms a triangle that also intersects the Philosopher's Stone [17, 18]. The location of the Philosopher's Stone is the same area as the zero-point crossover of the infinity symbol (called the Singularity or the Primal Point of Unity) in the Rodin Coil schematic, based on the nonagon and modular arithmetic [19], with parallels to Walter Russell's cosmogony [20]. The pentagon angle of $72^{\circ}$ minus $32^{\circ}$ is equal to the nonagon angle of $40^{\circ}$. The vertex angle of the nonagon, $140^{\circ}$, when considered as the central angle of a triangle in a circle forms the Egyptian hieroglyph for neb or gold [21] related to quintessence, or the "unified field" of physics.

The symbolic form of the Emerald Tablet is also described by Sir Laurence Gardner as the "Alchemical Medallion of the Hidden Stone" and he reports that Newton and Boyle's discoveries were attributed to help from the archive of the Hermetic Table [17, 18]. Also stated by John Michell: "Newton, who laid the foundations of modern cosmology, was also one of the last of the scholars of the old tradition who accepted that the standards of ancient science were higher than the nobler, and sought, like Pythagoras, to rediscover the ancient knowledge." [11]. From the symbolism of Pauli's World Clock $9 \times 9 \times 32=2592$, compared to the archetypal $144 \times 180=25,920$ of the Platonic Year completing a $360^{\circ}$ precessional cycle [7]. Also, 25,920/504 $=360 / 7 \simeq 85 / \sqrt{e}$, see Eq. (1), and $e$ is Euler's number, base of the natural logarithm. The proportion for the classical "squared circle" construction is $8 / 9=320 / 360$ and $8 \times 9=72=32+40$.

Pauli's interpretation of the World Clock as the "three permeating the four" is essential to the polemic between Fludd and Kepler that Pauli tried to resolve within himself and related to his presentation in "The Influence of Archetypal Ideas on the Scientific Theories of Kepler" of the hieroglyphic monad in Fludd's excerpt describing the quaternary $[3,22]$. Pauli's interpretation also relates to the Pythagorean-Egyptian tradition regarding the 3, 4, 5 right triangle that is the basis for the construction of the Cosmological Circle. The various interpretations of the Cosmological Circle include the maze of nested polyhedra within the dodecahedron and their transformations. David Lindorff comments, "Pauli's sense that number in itself had a deep psychological significance is striking; it would later become of singular importance to him. ... He wrote, 'Here new Pythagorean elements are at play, which can perhaps be still further researched."' [23]. Harald Atmanspacher and Hans Primas explain, "Pauli understood that physics necessarily gives an incomplete view of nature, and he was looking for an extended scientific framework." [24]. Pauli also worked with Marie-Louise von Franz, who wrote in Number and Time, "Numbers, furthermore, as archetypal structural constants of the collective 
unconscious, possess a dynamic, active aspect which is especially important to keep in mind. It is not what we can do with numbers but what they do to our consciousness that is essential." [25]. With this numerical analogy and parallels to neuroscience, Mark Morrison states in his overview, Modern Alchemy: "At this border of science and our deepest sense of our mental and even spiritual selves, alchemy is again demonstrating its relevance and durability." [26]. Other examples of solving the Kepler-Fludd problem that Pauli symbolized by the numbers three and four are found in the philosophy of Joseph Whiteman [27] and Franklin Merrell-Wolff [28].

\section{The fine-structure constant calculation}

The fine-structure constant has a dimensionless value determined by the most recent experimental-QED calculations: $\alpha^{-1}=137.035999173$ (35) (T. Aoyama, et al [29]) and $\alpha^{-1}=137.0359991733$ (344) (T. Kinoshita [30]). Approximating $\alpha^{-1} \simeq 137.035999$ 168:

$$
\sin \alpha^{-1} \simeq 504 / 85 \kappa \text {. }
$$

The quantitative and qualitative reasoning for the approximation is significant to Plato's geometry, $7 \times 72=111+393=504$, proportional to the large radius of the Cosmological Circle [31], and Plato's favorite $5040=7$ !, of the larger harmonic proportion [8]. $\cos (\pi / 16) \cot (\pi / 16) \simeq 504 / 85.6 \times 85=6+504.2 \times 54=108$ and $108+144=252$. $2 \times 252=504$. The polygon circumscribing constant [32], $\kappa \simeq 8.700036625208 \simeq$ $e^{2} \sec 32^{\circ}$ and $\cot 32^{\circ} \simeq \phi$. Another calculation involves Pythagorean triangles related to the Cosmological Circle and the prime constant [33], $\rho \simeq 0.414682509851111 \simeq \phi \sqrt{5} / \kappa$, which has a binary expansion corresponding to an indicator function for the set of prime numbers. The inverse fine-structure constant again:

$$
\alpha^{-1} \simeq 157-337 \rho / 7
$$

where $\alpha^{-1} \simeq 137.035999168$, approximately the same value as determined in Eq. (1) from above. The square of the diagonal of a "prime constant rectangle" is $1+\rho^{2} \simeq \kappa / e^{2} \simeq$ $5 / 3 \sqrt{2} \simeq \sec 32^{\circ}$, with the angle from "Pauli's triangle" found above. $180-23=157$, and $360-23=337.23+37=60,60 / \phi \simeq 37$, and $\phi \equiv(1+\sqrt{5}) / 2$. see [34]. $37+120=157$. $23+85=108$, proportional to the Moon radius of the Cosmological Circle. $7^{2}+$ $108=157$. Related to this is the main Pythagorean triangle 108, 144, 180. The triangles 85, 132, 157, and 175, 288, 337 are primitive Pythagorean triples. $60+72=132$ and $72+85=157.85+90=175,4 \times 72=288$, and $85+504 / 2=157+180=337.6^{2}+7^{2}=85$ and another triangle is $36,77,85$. 36 is the basic multiplier for the $3,4,5$ right triangle geometry, while 72 is the next. With the two basic radii 7 and $11,7 \times 11=77$, and $5 \times 36=180$.

Other related approximations include $1+\rho \simeq \sqrt{2}, \cos 32^{\circ} \simeq 2 \rho, 5 \rho \simeq \phi_{f} / \phi$ where $\phi_{f}$ is the reciprocal Fibonacci constant [34], and 360/ $\phi^{3} \simeq 85$. Also, $\alpha \simeq \rho / 32 \sqrt{\pi}$. The outer radius of the regular dodecahedron $(\sqrt{3}+\sqrt{15}) / 4 \simeq \gamma / \rho$ where $\gamma$ is the Euler-Mascheroni constant, see Eqs. (4) and (5). 
An "infinitely nested set of circumscribed polygons and circles" [32] gives the polygon circumscribing constant $\kappa \equiv \sec (\pi / 3) \sec (\pi / 4) \sec (\pi / 5) \ldots$, which is also formulated as a converging series involving the Riemann zeta function $\zeta(s)$ that is found in determining alpha. The Riemann zeta function is involved in the perturbative determination of the fine-structure constant from quantum electrodynamic theory and the experimentally measured value of the electron's gyromagnetic ratio [35].

In 1949 Wolfgang Pauli and Felix Villars published a paper together on Pauli-Villars regularization for the problem of infinities in quantum electrodynamics [36]. Pauli also corresponded with Julian Schwinger, noted here for his introduction of $\alpha / 2 \pi$ in the corrective calculation for the anomalous magnetic moment of the electron and his zeta function regularization for the renormalization effort.

Accurate to seven places, $\sin \alpha^{-1} \simeq 8 \delta / 7$, where $\delta$ is Gompertz constant (or the EulerGompertz constant, which can also be expressed in relation to the Euler's number [37]), $\delta \equiv-e \operatorname{Ei}(-1) \simeq \phi / e$, where $\mathrm{Ei}$ is the exponential integral. Returning to the polygon circumscribing constant, $\kappa^{2} \simeq 76, \kappa^{2}+\pi^{2} \simeq 85, \sin \alpha^{-1} \approx 32 \phi / \kappa^{2}$, and $\sin \alpha^{-1}$ is the approximate ratio of the $32^{\circ} \times \phi \simeq 51.8^{\circ}$ base angle of the Great Pyramid of Giza to the apex angle of approximately $76^{\circ}$. Vertex angle of the pentagon $108^{\circ}-32^{\circ}=76^{\circ}$ and $\csc \alpha^{-1} \simeq R \sqrt{\phi}$, see Eq. (4), with $R$ as the radius of the regular heptagon with side equal to one. $\alpha^{-1} \simeq 16 \pi^{2} / R$ and $R \simeq 2 \gamma \simeq-\ln (\rho \sqrt{\gamma})$, prime constant $\rho$ with $\gamma$, the Euler-Mascheroni constant; $\gamma \approx 5 / \kappa$. Also, $\kappa+D \simeq 11$, the basic diameter of the Cosmological Circle, where $D$ is the diameter of the regular heptagon with side equal to one [8]. The PDG [38] value for the strong coupling constant $\alpha_{s} \simeq 0.1184(7)$ is proportional: $\alpha_{s} / \alpha \simeq \kappa / \pi \rho^{2} . \alpha_{s} \simeq 1-\kappa / \pi^{2} \simeq \sec 32^{\circ} / \pi^{2} . \kappa \rho \phi^{2} \simeq \phi / \rho^{2}$ is the diagonal of a 5 by 8 approximate golden rectangle.

Brown Landone writes that long before the Egyptians the Teleois were used by the ancient masters of Tiajura, then the Tiahuanacans and Incas of South America. "Teleois numbers form the long lost canon of Polykleitos, since they were used to determine the structures of all great temples of Greece and Egypt where Pythagoras lived ..." [39]. "The Teleois proportions are used by the creative force because they best fit the electromagnetic energy fields of the atom." [40]. As part of a series based on modulo 3 arithmetic, the Teleois proportions are easily noticeable in the Queen's Chamber of the Great Pyramid of Giza, designed with seven Teleois spheres that also correspond to the geometry included in the Cosmological Circle. "Within the great sphere of 31 represented by a circle - six other Teleois circles exactly contact or intersect each other in perfect Teleois proportions." [39]. The diameters are 1, 4, 7, 10, 13, 19, and 31 . The sum of these seven diameters is 85 , harmonic of the $\sqrt{\alpha}$. The sum of the first six diameters is 54 .

William Conner also referenced the Teleois as a "cosmic formula behind form in the physical world" and modifies this series with a 144 multiplier beginning with 4 as 144, giving a culminating Teleois diameter of 11,664 or $108^{2}$ ("a number of extraordinary interlocking potential" determining the root tone generators of his Fibonacci-harmonic Quadrispiral and also found in the Great Pyramid proportions) [10]. 11,664 is also the proportional harmonic of $\alpha / 2 \pi$, equal to the classical electron radius divided by its Compton wavelength. 
Julian Schwinger introduced $\alpha / 2 \pi$ to the quantum electrodynamic problem of the anomalous magnetic moment of the electron, see Eq. (6) [41]. The harmonic of $\alpha / 2 \pi$ is also the value of Coral Castle builder Ed Leedskalnin's proportion, proclaimed by some of his followers as the "secret of the universe" [42]. On the Teleois again, "Understand the proportions of the atom and its electromagnetic frequencies and you can understand why the proportions of the Teleois were used." [40]. $\alpha^{-1} \simeq 85 \phi$ and $\csc \alpha^{-1} \simeq \sqrt{85} / 2 \pi$. More exactly, $\alpha^{-1} \simeq 137 \tan (\tanh (302 / 285))$ where $302 / 285=1+17 / 285 \simeq \sqrt{2 / e^{\gamma}} \simeq \delta \sqrt{\pi}$, see Eq. (4) discussion. From the harmonic braiding shown by William Conner [10], $370-285=85,5 \times 17=85,360-58=302$, and $32+58=90$.

From William Eisen's [43] construction of the "All-Seeing Eye" $60^{\circ}+77^{\circ}=137^{\circ}$ and $\sin 137^{\circ}=\cos 47^{\circ} \cdot 47^{\circ} / 2=23.5^{\circ}$, the approximate tilt of the Earth axis, considered by Scott Creighton and Gary Osborn in The Giza Prophecy as "the most important of the precessional angles encoded in the Giza pyramids ..." [44]. With tetrahedral angle, $19.5^{\circ}+23.5^{\circ}+47^{\circ}=90^{\circ}$. Additionally, the northern shaft in the King's Chamber pointed to Alpha Draconis, circa 2450 B.C., at an angle of approximately $32^{\circ}$. Corresponding to the construction of the Great Pyramid layering of stones and Pauli's World Clock symbolism is the connection between paramagnetism (including Pauli-paramagnetism) and the diamagnetism found in the golden ratio geometry of organic growth [45].

\section{Quintessential questions of the golden ratio}

A golden rectangle of whirling squares constructed by Bonnie Gaunt, based on the sacred geometry of the solar system, has the side of a square proportional to 504 [31]. $504 / 85 \simeq \sqrt{2} \phi^{3} \approx 2 \sqrt{\kappa}$. Dan Winter puts two of these golden rectangles together in opposite directions, constructs a Vesica Piscis, and draws two intersecting golden ratio spirals. This forms a main Pythagorean triangle $(\phi, \phi+1, \phi \sqrt{\phi+2})$ with side ratios of $\phi \sqrt{\phi+2} / \phi=\sqrt{\phi+2} \approx \csc 32^{\circ}$ which is also the approximate cube/sphere ratio of $6 / \pi=\pi / \zeta(2)$; a significant ratio in ancient Egyptian geometry [34]. Also shown above, $\cot 32^{\circ} \simeq \phi$.

Continuing with Winter's geometric construction, the golden ratio spirals are then shown dynamically forming a toroidal vortex that fits in a dodecahedron. Winter suggests this action is the source of gravity and the formation of mass, by "fractal charge compression" (charge is interpreted broadly here as a form of the ether, or the alchemical quintessence, in which Winter and others report an interesting relationship with the dodecahedral structure of the noble elements). The dodecahedron has been considered a three-dimensional version of the Cosmological Circle and was associated with the alchemical quintessence $[8,34]$. Also suggestive, the expression for Newton's gravitational constant $G$ in terms related to Planck momentum is $G=\hbar c / m_{P}^{2}$ and from the relation for the fine-structure constant $\hbar c=e^{2} / \alpha$ therefore, $\alpha G=e^{2} / m_{P}^{2}$, square of the electric charge to Planck mass ratio [34, 46].

In William Eisen's Cabala the number 137 is interpreted as the MG or "image" and 37 is the CG or "center of gravity" [43]. According to Marcus Chown [47], "Perhaps the most surprising place the golden ratio crops up is in the physics of black holes, a discovery made 
by Paul Davies," [48, 49] (for other examples see [50]-[53]). This brings up one of the most fundamental concepts of alchemy, the prima materia, or "first matter" symbolized by the color black (Pauli's blackbird) and related to the aethereal quintessence of the Philosopher's Stone.

The angular momenta found in massive particles are related to quintessence, the Pythagorean harmonic of Planck momentum as described by Malcolm Macleod, $Q^{2} \simeq$ $m_{P} c / 2 \pi$ [46], with the fundamental geometry related to the regular heptagon (which is related to the origin of calculus and the least action principle $[8,34])$. The quintessence $Q$ has a Pythagorean relationship with $C$ :

$$
C^{2}=Q^{2}+Q^{2} \simeq \cot (\pi / 7),
$$

where $C^{2}$ is the approximate inner diameter of the regular heptagon with side equal to one. Quintessence also has other simple relations with the pentagon geometry, $\tan 72^{\circ} \simeq$ $1+2 Q^{2}$, and $Q \simeq \sqrt{3} \cos 54^{\circ} \simeq 2 \tan 27^{\circ}$ (54 is half the vertex angle of the pentagon and also noted, $\ln 32 \simeq 2 \sqrt{3}$ ). $C$ is also a fundamental harmonic of the "grid speed of light" found in the World Grid [11,34], the fundamental tone of William Conner's version of the Pythagorean Table [10], and proportional to the decagon vertex angle. Also, $2 C^{2} \simeq \sqrt{e^{2}+\pi^{2}}$, and $C^{2} \simeq 5 \rho \simeq \phi_{f} / \phi$ where $\phi_{f}$ is the reciprocal Fibonacci constant [34]. $Q^{2} \simeq 25 / 24$, canonical ratio of ancient metrology related to the precessional cycle calculation [11]. The quintessence harmonic related to the golden ratio and inverse fine-structure constant:

$$
\csc \alpha^{-1} \simeq C Q=\sqrt{2} Q^{2} \simeq 2 \gamma \sqrt{\phi}
$$

$\cos 32^{\circ} \simeq \gamma C Q$ and $R \simeq 2 \gamma$ with $R$ as the outer radius of the regular heptagon with side equal to one. Thus the fine-structure constant is shown related to the angular momentum property characteristic of massive particles, which again is related to "Pauli's triangle." Other approximations of quintessence: $Q \simeq 1+\alpha \phi^{2} \simeq \sqrt{2} \rho / \gamma$, where $\gamma$ is the EulerMascheroni constant again. Also, $Q \simeq \ln (\kappa / \pi) \simeq \ln (R / \rho)$ and $Q / 3 \simeq \sqrt{\gamma / 5} \simeq e / 8$. $Q^{3} \simeq$ $\sqrt{2 / e^{\gamma}} \simeq \sqrt{\phi / C}$. With the inverse of $\alpha / 2 \pi$ from Schwinger, in radians $\sin (2 \pi / \alpha) \simeq Q / \phi$.

Another geometric relationship, recalling Euler's formula shows a connection between the golden ratio, the dodecahedron, and quintessence: $e^{i Q} \simeq\left(\phi^{2} / 5\right)+\left(\sqrt{5} i / \phi^{2}\right) \simeq \cos Q+$ $i \sin Q$, thus $Q \simeq 1.019$ radians. The $\cos ^{-1}\left(\phi^{2} / 5\right) \simeq 58^{\circ}, \sin ^{-1}\left(\sqrt{5} / \phi^{2}\right) \simeq 59^{\circ}$ which shows angles close to the complementary angle in "Pauli's triangle" and the sum of both is approximately the regular dodecahedron dihedral angle.

The proton/electron mass ratio $m_{p} / m_{e} \simeq 1836.12567247$ [38] and with nine place accuracy $\csc \left(m_{p} / m_{e}\right) \simeq \sqrt{3}-\pi / 85$. Other important mass ratios also have simple approximations involving quintessence and "Pauli's triangle." The $\mathrm{W}$ boson from electroweak theory and the Higgs boson, $m_{w} / m_{H^{\circ}} \simeq Q \tan 32^{\circ}$. The $\mathrm{Z}$ boson from electroweak theory and the Higgs boson,

$$
m_{z} / m_{H^{\circ}} \simeq \cos ^{2} 32^{\circ} \simeq Q / \sqrt{2} .
$$

Also, the Higgs boson and the top quark from quantum chromodynamics, $m_{H^{\circ}} / m_{t} \simeq$ $Q \cos ^{2} 32^{\circ}$ [38] In later graphical representations Winter shows how racheting a cube five times by $32^{\circ}$ forms the dodecahedron, and explains how this is related to the opening of 
the pineal gland. The main supporting reference is the $32^{\circ}$ head tilt of the Great Sphinx of Giza noted by John Anthony West.

The aspect of the dodecahedron in question here is related to Krsanna Duran's Timestar, where Winter finds the square root of phi to be significant in the proportional relation between the dodecahedron and the rotation of the enclosed tetrahedra (recall the discussion above, $\cos 23.5^{\circ} \simeq \sqrt{\phi} \tan 36^{\circ}$ showing the decagon angle and $\phi=2 \cos 36^{\circ}$ ) [54]. The action has parallels to Pauli's vision of a World Clock described by Jung as a mandala.

The configuration of polygons determining the polygon circumscribing constant $\kappa$ was also described as a mandala, notable in the ancient canon according to Marie Franz and Elson Haas [25]. The harmonic of the polygon circumscribing constant is also found in the diameter of Stonehenge (with an architectural design also based on the Cosmological Circle) and 87/54 $\phi$. Also from the Cosmological Circle, the Earth-Moon radius divided by the Earth radius, 504/396 $\simeq \sqrt{\phi} \simeq 108 / 85$, with the proportional Moon radius of 108 [31, 34].

A more precise approximation for quintessence reveals a factor of 7 and 85 again, $Q \simeq \exp (42 /(56 P+85 e))^{2}[8,34] . P \simeq 1.32479572447 \simeq 5 Q^{3} / 4$, the silver number $P[8]$ is the root of $x^{3}-x-1=0$, also the limiting ratio of successive terms in the Padovan sequence and Perrin sequence. The silver number has a property similar to the golden ratio, $P+1=P^{3}$.

\section{Electron magnetic moment anomaly}

In modern quantum electrodynamics the g-factor of the electron is represented as a series expansion in powers of $\alpha / 2 \pi$ yielding the value $g / 2 \simeq 1.00115965218073$ (28) [35], also one of the most precise experimental measurements [35]. A general approximation of the anomalous magnetic moment of the electron $a_{e} \equiv(g-2) / 2$ can be made from explanations of spin-orbit coupling and reasoning from alternative theory [45], [55]-[58]:

$$
g / 2 \simeq 1+(\alpha / 2 \pi)-(\phi \alpha / 2 \pi \sqrt{2})^{2}+(\alpha / 2 \pi \gamma)^{3}-(\alpha / 2 \pi \phi)^{4} .
$$

$g / 2 \simeq 1.00115965218075$ with the value of alpha from Eqs. (1) and (2). The harmonic formula above involves a convergence of Julian Schwinger's work with Green's functions [41], the classical geometry behind Pauli's World Clock vision, and the corresponding golden ratio $\phi$ aspect of the Cosmological Circle (from the center to the mid-edge of the dodecahedron equals $\phi^{2} / 2=\pi$ /golden angle in radians and the chord of the pentagon face is $\phi$ ). From the regular heptagon, the radius with side equal to one, $R \simeq 2 \gamma$. The Euler-Mascheroni constant is the finite part of the harmonic series in the Riemann zeta function of $\zeta(1)$ [59], used in quantum electrodynamic calculations [60, 61]. Euler's constant $\gamma \simeq 0.577215664901532$ [62], for $\gamma$ and the Riemann zeta function $\zeta(s)$ see Julian Havil's Gamma [62]. The heptagon of the Cosmological Circle is related to the torus. With volume of a torus, or the hypersphere surface area: $2 \pi^{2} R^{3} \simeq 32 R^{3} / \phi$, and $R=\phi \sec 32^{\circ}, \alpha^{-1} \simeq 2 \pi^{2}\left(\phi \sec 32^{\circ}\right)^{3} \cdot R^{3} \simeq 360^{\circ} / \tan ^{-1}(\sqrt{\phi})$, with an approximate heptagon angle. The cube/sphere ratio, $6 / \pi \simeq \phi \sec 32^{\circ}$. 
As Malcolm Mac Gregor explains in The Power of Alpha, the essence of the finestructure constant is the ratio of "... the 'spherical geometry' of the electrostatic energy $E=e^{2} / r_{e}=m c^{2}$ to the 'spherical geometry' of mechanical energy, $\hbar c / r_{m}=m c^{2}$, which is defined by the Compton radius of a relativistically spinning sphere." [63]. Then $r_{e} / r_{m}=e^{2} / \hbar c=\alpha$.

Another interpretation of the fine-structure constant related to Pauli's World Clock geometry is the standard perspective of action, product of energy and time. Two elementary particles separated by a distance $r$ have an electrostatic energy of $e^{2} / r$ and the time for light to travel a distance $r$ is $r / c$, so the action is $\left(e^{2} / r\right) \times(r / c)=e^{2} / c$. Since the unit of quantum action for light is $\hbar$ (from $E=\hbar \omega$ ), the ratio of the electrical action to the quantum action is $e^{2} / \hbar c=\alpha$.

\section{Conclusions}

Since Arnold Sommerfeld's introduction of the fine-structure constant and the discovery of Planck's constant by blackbody radiation, attempts were made to find relationships between them. They were found connected by prime numbers, particular values of the Riemann zeta function, the Boltzmann constant, and even a dimensionless blackbody radiation constant [64]. Pauli displayed elegance in his own mathematical techniques for calculating the hydrogen spectrum [65].

From an overview by Domenico Giulini, "In his last paper on the subject of discrete symmetries ... Pauli comes back to the question which bothered him most: how is the strength of an interaction related to its symmetry properties?" [66]. This question is associated with the manifestation of charge from alchemical quintessence, the rotations associated with the symmetry groups of Platonic solids, and part of the archetypal process behind Pauli's World Clock.

In "Science and Western Thought" from Wolfgang Pauli: Writings on Philosophy and Physics Pauli asks the question, "Shall we be able to realize, on a higher plane, alchemy's old dream of psychophysical unity, by the creation of a unified conceptual foundation for the scientific comprehension of the physical as well as the psychical?" [67]. Kalervo Laurikainen responds in The Message of the Atoms, "If we are not bound to materialistic presuppositions, the psycho-physical unus mundus of Pauli and Jung appears, in fact, to be the natural ontology of quantum mechanics." [68]. This leads to a complementary representation of reality similar to Bohr's philosophy, rather than that of Cartesian dualism.

Thus Wolfgang Pauli considered, "Concepts created by mathematics, such as Riemann's surfaces, lend themselves very well to a symbolic representation of the relativization of the concept of time ...." and concluded that "... most modern physics lends itself to the symbolic representation of psychic processes." [16]. Pauli's World Clock vision involves the symbolic images and archetypal stages of alchemical transformation at the root of both mental and physical worlds [69].

From Pauli's imaginal geometry the unit circle on the complex plane was his $i$ ring, represented by $e^{i \theta}$, and yielding the "Pauli triangle" for $\theta \simeq Q$, quintessence in radians. 
For Pauli the ring embraced both intellect and intuition symbolic of the unus mundus.

The World Clock geometry combined with the self-recursive property enabled by the golden ratio and an alchemical interpretation of its symbolism is also descriptive of the pine cone shaped energy field of an activated pineal gland, often depicted in ancient Egyptian art along with the legendary bennu bird that is known for its association with the cycles of time and the final phase of the alchemical process (an awakening of the "golden" crown chakra in the aetheric field of mfkzt [17]). The pineal gland biology is an active area of research in modern biophysics and pineal activation might explain the "Pauli effect" reported by experimental physicists. Pauli knew of Descartes ideas about the pineal gland and Plato suggested that mathematical discipline such as Pauli engaged in could help awaken this special gland. Pauli wrote several unpublished papers considering the symbolic representation of mental process from the mathematics of the electromagnetic field. From the geometry of neb, the hieroglyph for gold [21], $Q \simeq 140 \alpha$. From the previous force field analogy with the four basic constants of "recovered science," $Q \simeq \pi e^{2} / \kappa \phi^{2}$ [34]. From the torus, $\tan ^{-1}(\sqrt{\phi})$ is the approximate heptagon angle, with $\sqrt{\phi} \simeq 4 / \pi$.

Beyond the alchemical interpretations of Carl Jung, the torus topology that relates the dynamics of Einstein's relativity with classical mechanics and its geometry is found in Egyptian art, especially their representation of anatomical proportions. Some depictions of Tehuti-Thoth from ancient Egyptian art are similar to Pauli's World Clock having the bird space-form of the alchemical tradition with the archetypal signs and symbols of time-cycles [70]-[72].

In The Sphinx and the Rainbow David Loye states, "Wolfgang Pauli felt there could be a correspondence between the wave-particle mystery in physics and the mind-body problem in philosophy ..." [73]. F. David Peat also writes in Synchronicity, "Pauli, as a physicist, was also seeking to discover an inner unity between the elementary particles and their abstract symmetries." [74].

Beverley Zabriskie writes in her introduction to Atom and Archetype, the Pauli/Jung letters edited by C.A. Meier, "For Pauli, symmetry was the archetypal structure of matter. Just as the alchemists looked for the substratum of reality beneath matter, he came to the view that the elementary particles were not themselves the ultimate level of realty."[75]. Then she explains, "As he became more familiar with alchemy as a psychophysical unity, Pauli saw the same lumen naturae, the light of nature, or the "spirit in matter,' glimpsed by Paracelsus and Jung." [75].

Writing on "Wolfgang Pauli's Philosophical Outlook" in Across the Frontiers, Werner Heisenberg says; "Among the studies to which Pauli was prompted by the philosophical labors just referred to, it was those on the symbolism of the alchemists which left particularly lasting traces behind ..." [76]. Many of Pauli's ancestors were from Prague, a traditional center for alchemical activity [6].

In an article about Pauli by his former assistants Marcus Fierz and Victor Weisskopf, "If we ask ourselves what above all was Pauli's calling the answer would be: he was a natural philosopher in the classical sense of the phrase - as it applies to Kepler, Galileo, and Newton." [77]. A later retrospective by Victor Weisskopf shows Pauli "... developed a deep friendship with Gershon Scholem, the great scholar and world authority on Jewish 
mysticism, the Kabalah. (The Kabalah ascribes a number to each word of the Hebrew language, a number that has a deep symbolic significance. The number corresponding to the word Kabalah happens to be 137.)" [78].

In his essay, "Pauli (Wolfgang) 1900-1958," Charles Enz (Pauli's last assistant) writes; "This number 137 symbolized for Pauli the link with the magic world of the alchemists which has so much fascinated him." [79]. Recalling his preoccupation with the finestructure constant and research on synchronicity with Carl Jung, Pauli was moved upon finding his room number was 137 at the Red Cross hospital during his last days; when pineal activation can happen in transition [16, 79].

\section{Acknowledgments}

Special thanks to Case Western Reserve University, Franklin Merrell-Wolff Fellowship,

Social Science Research Network, Pauli Archives, MathWorld, and WolframAlpha.

\section{References}

[1] Enz, C.P. No Time to be Brief: A Scientific Biography of Wolfgang Pauli, Oxford University Press, New York: Oxford University Press, 2002.

[2] Miller, A.I. Deciphering the Cosmic Number: The Strange Friendship of Wolfgang Pauli and Carl Jung, New York: W.W. Norton, 2009.

[3] Enz, C.P. \& Meyenn, K. eds. Wolfgang Pauli, New York: Springer, 1994.

[4] Peierls R. "Wolfgang Ernst Pauli, 1900-1958," Biographical Memoirs of Fellows of the Royal Society, 5, 174-92 (1960).

[5] Gustafson, J.R. "Wolfgang Pauli 1900 to 1930: His Early Physics in Jungian Perspective," (2010 ) arXiv:1003.3223.

[6] Várlaki, P. et al, "Historical Origin of the Fine Structure Constant, Parts I-III," Acta Polytechnica Hungarica, 7, 1, 119-157 (2010) 8, 2, 161-196; 8, 6, 43-78 (2011).

[7] Brumbaugh, R.S. Plato's Mathematical Imagination: The Mathematical Passages in the Dialogues and Their Interpretation, Indiana: Indiana University Press, 1954.

[8] Sherbon, M.A. "Nature's Information and Harmonic Proportion," SSRN Philosophy of Science eJournal, 5, 3 (2012) abstract=1766049.

[9] Jung, C.G. Mysterium Coniunctionis, London: Routledge \& Kegan Paul, 1970.

[10] Conner, W.B. Harmonic Mathematics, Chula Vista, CA: Tesla Book Co. 1982.

[11] Farrell, J.P. \& Hart, S.D. The Grid of the Gods: The Aftermath of the Cosmic War and the Physics of the Pyramid Peoples, Kempton: Adventures Unlimited, 2011.

[12] Jung, C.G. Psychology and Alchemy, London: Routledge \& Kegan Paul, 1970. 
[13] Seifer, M. Transcending the Speed of Light, Rochester, VT: Inner Traditions, 2008.

[14] Meier, C.A. ed. Atom and Archetype: The Pauli/Jung Letters 1932-1958, Princeton, NJ: Princeton University Press, 2001, p.59.

[15] Jung, C.G. \& Pauli, W. The Interpretation of Nature and the Psyche, London: Routledge \& Kegan Paul, 1955.

[16] Pauli, W. "Modern Examples of Background Physics," in Meier, C.A. ed. Atom and Archetype, Princeton, New Jersey: Princeton University Press, 2001.

[17] Gardner, L. Genesis of the Grail Kings, London: Bantam Press, 1999.

[18] Dobbs, B.J. "Newton's Commentary on the Emerald Tablet of Hermes Trismegistus," in Merkel, I. \& Debus A.G. Hermeticism and the Renaissance: Intellectual History and the Occult in Early Modern Europe, Washington: Folger, 1988.

[19] Rodin, M. "Vortex Based Mathematics: Basis for the ExtraOrdinary Rodin Coil," ExtraOrdinary Science \& Technology, Jan/Feb/Mar, 1-18 (2010).

[20] Russell, W. The Secret of Light, Virginia: University of Science \& Philosophy, 1974.

[21] Gadalla, M. Egyptian Harmony, Greensboro: Tehuti Research Foundation, 2000.

[22] Pauli, W. "The Influence of Archetypal Ideas on the Scientific Theories of Kepler," in Enz, C.P. \& Meyenn, K. eds. Wolfgang Pauli: Writings on Physics and Philosophy, New York: Springer, 1994, pp.219-279.

[23] Lindorff, D. Pauli and Jung, Wheaton, IL: Quest Books, 2004, p.143.

[24] Atmanspacher, H. \& Primas, H. eds. Recasting Reality: Wolfgang Pauli's Philosophical Ideas and Contemporary Science, New York: Springer, 2008, p.2.

[25] Franz, M.L. Number and Time: Reflections Leading Toward a Unification of Depth Psychology and Physics, Evanston: Northwestern University Press, 1974, p.65.

[26] Morrisson, M.S. Modern Alchemy, Oxford: Oxford University Press, 2007, p.191.

[27] Halsbury, L. "Professor Whiteman's Philosophy of Space and Time," Philosophy, 45, 171, 61-65 (1970).

[28] Merrell-Wolff, F. Transformations in Consciousness: The Metaphysics and Epistemology, Albany, NY: State University of New York Press, 1995.

[29] Aoyama, T., Hayakawa, M., Kinoshita, T. \& Nio, M. "Tenth-Order QED Contribution to the Electron g-2 and an Improved Value of the Fine Structure Constant," Physical Review Letters, 109, 111807 (2012) arXiv:1205.5368v2.

[30] Kinoshita, T. "Fine Structure Constant Derived from the Electron g-2 with the QED Theory Up to the Tenth-Order," in Precision Electromagnetic Measurements (CPEM), Ithaca, New York: IEEE Conference Publications, 2012, pp.242-243. 
[31] Gaunt, B. Beginnings: The Sacred Design, Kempton: Adventures Unlimited, 2000.

[32] Weisstein, E.W. "Polygon Circumscribing," From MathWorld - A Wolfram Web Resource, www.mathworld.wolfram.com/PolygonCircumscribing.html.

[33] Weisstein, E.W. "Prime Constant," From MathWorld - A Wolfram Web Resource, www.mathworld.wolfram.com/PrimeConstant.html.

[34] Sherbon, M.A. "Mathematical Constants of Natural Philosophy," SSRN History of Western Philosophy eJournal, 5, 2 (2012) abstract=1646568.

[35] Hanneke, D., Fogwell, S. \& Gabrielse, G. "New Measurement of the Electron Magnetic Moment and the Fine Structure Constant," Physical Review Letters, 100, 120801 (2008) arXiv:0801.1134v2.

[36] Pauli, W. \& Villars, F. "On the Invariant Regularization in Relativistic Quantum Theory," Reviews of Modern Physics, 21, 434-444 (1949).

[37] Finch, S.R. "Euler-Gompertz Constant," Mathematical Constants, Cambridge: Cambridge University Press, 2003, p.424.

[38] Beringer, J. et al (Particle Data Group), Physical Review D86, 010001 (2012).

[39] Landone, B. Prophecies of Melchi-Zedek in the Great Pyramid and the Seven Temples, New York: The Book of Gold, 1940, p.141.

[40] Hardy, D. et al, Pyramid Energy, Clayton, GA: Cadake Industries, 1987.

[41] Schwinger, J. "On Quantum-Electrodynamics and the Magnetic Moment of the Electron," Physical Review, 73, 416 (1948) in Schwinger, J. ed. Selected Papers on Quantum Electrodynamics, New York: Dover Publications, 2003.

[42] McClure, R. \& Heffron, J. Coral Castle: The Mystery of Ed Leedskalnin and his American Stonehenge, Ohio: Ternary Publishing, 2009.

[43] Eisen, W. The Essence of the Cabalah, Camarillo, CA: DeVorss \& Company, 1984.

[44] Creighton, S. \& Osborn, G. The Giza Prophecy, Rochester, VT: Bear \& Co., 2012.

[45] Winter, D. One Crystal's Dance - The Star Mother: Geometric Keys to the Resonant Spirit of Biology, Eden, NY: Crystal Hill Farm, 1987, p.20.

[46] Macleod, M.J. "Quintessence-Momentum as Link Between Mass and Charge," (2011) viXra:1102.0032.

[47] Chown, M. "The Golden Rule: It Links Art, Music and Even Architecture," The Guardian, Wednesday, January 15 (2003).

[48] Davies, P.C.W. "Thermodynamic Theory of Black Holes," Reports on Progress in Physics, 41, 1313 (1979). 
[49] Davies, P.C.W. "Thermodynamic Phase Transitions of Kerr-Newman Black Holes in de Sitter Space," Classical and Quantum Gravity, 6, 1 (1989).

[50] Nieto, J.A. "A Link Between Black Holes and the Golden Ratio," (2011) arXiv:1106.1600v1.

[51] Koca, M. \& Koca, N.O. "Radii of the $\mathrm{E}_{8}$ Gosset Circles as the Mass Excitations in the Ising Model," (2012) arXiv:1204.4567v2.

[52] Heyrovska, R. \& Narayan, S. "Fine-Structure Constant, Anomalous Magnetic Moment, Relativity Factor, and the Golden Ratio that Divides the Bohr Radius," (2005) arXiv:physics/0509207v1.

[53] Keen, J.S. "The Mind, Intergalactic Space, and Phi," NeuroQuantology, 9, 4, 649659 (2011) viXra:1106.0051.

[54] Duran, K. The Human Odyssey, Bloomington, IN: Trafford Publishing, 2012.

[55] Dennis, L., Gray, R., Kauffman, L., McNair, J. \& Woolf, N. "A Framework Linking Non-living and Living Systems," Foundations of Science, 14, 3, 217-238 (2009).

[56] Holverstott, B.G. "Quantum Electrodynamics," in Scientific Realism, Empiricism, and Quantum Theory, Portland, OR: Reed College, 2007.

[57] Westgard, J.B. "Classical Modes of the Electron," in Electrodynamics: A Concise Introduction, New York: Springer, 1997, pp.405-414.

[58] Miller, A.I. Early Quantum Electrodynamics: A Source Book, Cambridge: Cambridge University Press, 1995.

[59] Zeidler, E. Quantum Field Theory II, New York: Springer, 2008, p.55.

[60] Drell, S.D. \& Zachariasen, F. "Form Factors in Quantum Electrodynamics," Physical Review, 111, 6, 1727-1735 (1958).

[61] Drell, S.D. \& Pagels, H.R. "Anomalous Magnetic Moment of the Electron, Muon, and Nucleon," Physical Review, 140, 2B, 397-407 (1965).

[62] Havil, J. Gamma: Exploring Euler's Constant, Princeton, NJ: Princeton University Press, 2003.

[63] Mac Gregor, M.H. The Power of Alpha, Hackensack, NJ: World Scientific, 2007.

[64] Xiao, K. "Dimensionless Constants and Blackbody Radiation Laws," Electronic Journal of Theoretical Physics, 8, 25, 379-388 (2011).

[65] Valent, G. "The Hydrogen Atom in Electric and Magnetic Fields: Pauli's 1926 Article," American Journal of Physics, 71, 2, 171-175 (2003). 
[66] Giulini, D. "Concepts of Symmetry in the Work of Wolfgang Pauli," in Atmanspacher, H. \& Primas, H. eds. Recasting Reality: Wolfgang Pauli's Philosophical Ideas and Contemporary Science, New York: Springer, 2008, pp. 33-82.

[67] Pauli, W. "Science and Western Thought," in Enz, C.P. \& Meyenn, K. eds. Wolfgang Pauli: Writings on Physics and Philosophy, New York: Springer, 1994, pp.137-148.

[68] Laurikainen, K.V. The Message of the Atoms: Essays on Wolfgang Pauli and the Unspeakable, New York: Springer, 1997, p.41.

[69] Wolf, F.A. "Towards a Quantum Field Theory of Mind," NeuroQuantology, 9, 3, $442-458$ (2011).

[70] Wilcock, D. The Source Field Investigations, New York: Dutton, 2011.

[71] Gieser, S. The Innermost Kernel: Depth Psychology and Quantum Physics: Wolfgang Pauli's Dialogue with C.G. Jung, Berlin: Springer, 2005.

[72] Cox, B. "Egyptian and Form Energy Head-Dress of the Ancients," Santa Barbara, CA: Life Understanding Foundation, 1979.

[73] Loye, D. The Sphinx and the Rainbow, New York: Bantam, 1983, p.238.

[74] Peat, F.D. Synchronicity: The Bridge Between Matter and Mind, Toronto, NewYork: Bantam Books, 1987, p.20.

[75] Zabriskie, B. "Jung and Pauli," in Meier, C.A. ed. Atom and Archetype: The Pauli/Jung Letters 1932-1958, Princeton, NJ: Princeton University Press, 2001.

[76] Heisenberg, W. "Wolfgang Pauli's Philosophical Outlook," in Across the Frontiers, New York: Harper and Row, 1974, pp.30-38.

[77] Fierz, M.E. \& Weisskopf, V.F. "In Commemoration of Wolfgang Pauli," Physics Today, 12, 7, 16 (1959).

[78] Weisskopf, V.F. "Personal Memories of Pauli," Physics Today, 38, 12, 36 (1985).

[79] Enz, C.P. Of Matter and Spirit, Hackensack, NJ: World Scientific, 2009, p.95. 